\title{
The contribution of cardio-metabolic risk factors to estimated glomerular filtration rate (eGFR) decline in Indigenous Australians with and without albuminuria- the eGFR follow-up study.
}

Running title: Cardio-metabolic risk factors and eGFR decline

Elizabeth Barr ${ }^{1,2}$; Federica Barzi ${ }^{1}$; Jaquelyne T Hughes ${ }^{1,3}$; George Jerums ${ }^{4,5}$; Kerin O’Dea ${ }^{6}$; Alex Brown $^{7,8}$; Elif I Ekinci ${ }^{1,4,5}$ Graham RD Jones ${ }^{9,10}$; Paul D Lawton ${ }^{11}$; Ashim Sinha ${ }^{12}$; Richard J MacIsaac ${ }^{5,13}$; Alan Cass ${ }^{14}$ and Louise J Maple-Brown ${ }^{1,3}$

1. Menzies School of Health Research, Wellbeing and Preventable Chronic Disease Division, Darwin, Darwin, Australia

2. Baker Heart and Diabetes Institute, Clinical and Population Health, Melbourne, Australia

3. Royal Darwin Hospital, Division of Medicine, Darwin, Australia

4. Austin Health, Department of Endocrinology, Melbourne, Australia

5. University of Melbourne, Department of Medicine, Melbourne, Australia

6. University of South Australia, Nutrition and Population Health, Adelaide, Australia

7. University of South Australia, Aboriginal Health, Sansom Institute Health Research Operations, Adelaide, Australia.

8. South Australian Health and Medical Research Institute, Indigenous Health, Adelaide, Australia

9. St Vincent's Hospital, SydPath, Sydney, Australia

10. University of New South Wales, Department of Medicine, Sydney, Australia

11. Menzies School of Health Research, Epidemiology and Health Systems, Darwin, Australia

12. Cairns Base Hospital, Diabetes and Endocrinology, Cairns, Australia

13. St Vincent's Hospital Melbourne, Department of Endocrinology and Diabetes, Melbourne, Australia

14. Menzies School of Health Research, Director, Darwin, Australia.

Corresponding Author and Institution where work carried out:

Elizabeth Barr

Menzies School of Health Research

PO Box

Casuarina $0811 \mathrm{NT}$

Australia

Phone: +61385321827

Fax: + 61385321100

e-mail: Elizabeth.barr@menzies.edu.au

Journal: Nephrology

Word: 4364 ( $\max 4000$ including the abstract, text and references.)

Tables: 3 (Max 8 tables or figures)

Figure: 1

This is the author manuscript accepted for publication and has undergone full peer review but has not been through the copyediting, typesetting, pagination and proofreading process, which may lead to differences between this version and the Version of Record. Please cite this article as doi: $10.1111 /$ nep.13073 
References: $23(30 \max )$

This article is protected by copyright. All rights reserved. 


\begin{abstract}
Background: Risk factors for estimated glomerular filtration rate (eGFR) decline beyond albuminuria are not fully understood in Indigenous Australians who have a 6-fold risk of end-stage kidney disease. We assessed associations between cardio-metabolic risk factors and eGFR decline according to baseline albuminuria status to identify potential treatment targets.
\end{abstract}

Methods: The eGFR Follow-up study is a longitudinal cohort of 520 Indigenous Australians. Linear mixed regression was used to estimate associations between baseline cardio-metabolic risk factors and annual Chronic Kidney Disease Epidemiology Collaboration (CKD-EPI) eGFR change $\left(\mathrm{ml} / \mathrm{min} / 1.73 \mathrm{~m}^{2} /\right.$ year), among those classified with baseline normoalbuminuria (uACR $<3 \mathrm{mg} / \mathrm{mmol}$; $\mathrm{n}=297$ ), microalbuminuria (uACR 3-30mg/mmol; $\mathrm{n}=114$ ) and macroalbuminuria (uACR e30mg/mmol; $\mathrm{n}=109)$.

Results: After a median of 3.0 years of follow-up, progressive declines of the age- and sex-adjusted mean eGFR was observed across albuminuria categories (-2.0 [-2.6 to-1.4], -2.5 [-3.7 to -1.3] and $6.3[-7.8$ to -4.9$] \mathrm{ml} / \mathrm{min} / 1.72 \mathrm{~m}^{2} /$ year). Although a borderline association was observed between greater baseline $\mathrm{HbA}_{1 \mathrm{c}}$ and eGFR decline in those with macroalbuminuria $(\mathrm{p}=0.059)$, relationships were not significant in those with microalbuminuria $(p=0.187)$ or normoalbuminuria $(p=0.23)$. Greater baseline blood pressure, C-reactive protein, waist-to-hip ratio and lower HDL cholesterol showed non-significant trends with greater eGFR decline in the presence of albuminuria.

Conclusion: This study demonstrated that in a three year period marked eGFR decline was observed with greater baseline albuminuria. Cardio-metabolic risk factors were not strong predictors for eGFR decline in Indigenous Australians without albuminuria. Longer follow-up may elucidate the role of 
these predictors and other mechanisms in CKD progression in this population.

\section{Words: 245}

Keywords: albuminuria, diabetes mellitus, chronic kidney disease (CKD), haemoglobin $\mathrm{A}_{1 \mathrm{c}}$, Indigenous, risk factors

\section{Short Summary}

In this short follow-up longitudinal observational study of Indigenous Australians, marked estimated glomerular filtration rate (eGFR) decline was observed with greater baseline albuminuria. Cardiometabolic risk factors were not strong predictors for eGFR decline in Indigenous Australians without albuminuria. Longer follow-up may elucidate the role of these predictors and other mechanisms in chronic kidney disease progression in this population.

This article is protected by copyright. All rights reserved. 


\section{Introduction}

Chronic kidney disease (CKD) is a significant public health problem for Indigenous populations ${ }^{1,2}$. In Indigenous Australians, the risk of end-stage kidney disease (ESKD) is estimated to be six-fold higher compared to other Australians ${ }^{1}$. CKD is associated with diabetes, hypertension and cardiovascular disease, and is underpinned by social disadvantage, poverty and rapid change in diet and lifestyle ${ }^{3,4}$. Estimated glomerular filtration rate and albuminuria predict CKD progression and ESKD independent of each other, and of hypertension, diabetes, cholesterol and smoking 5 . However, there is little published literature in this field pertaining to Indigenous Australians. Despite a high prevalence of diabetes ${ }^{2,6}$, renal biopsy studies of Indigenous Australians have also demonstrated that CKD may not necessarily be attributed to diabetic nephropathy ${ }^{7}$, but to an array of cardio-metabolic factors, including hyperglycaemia in the pre-diabetes range, obesity, dyslipidaemia, hypertension and chronic inflammation that may arise from early life experiences such as low birth weight and infections ${ }^{4}$.

Few longitudinal studies have characterised the predictors of CKD progression in Indigenous populations ${ }^{8}$. In one remote Northern Australian community, albuminuria was the dominant predictor of eGFR decline ${ }^{9}$. Our recent study of a geographically diverse Indigenous Australian cohort at high risk for ESKD confirmed this association between albuminuria and CKD progression and outcomes for all levels of baseline eGFR. However, associations of central obesity, dyslipidaemia, hyperglycaemia, hypertension and C-reactive protein with CKD were not significant, possibly due to attenuation by the overwhelming strength of association between albuminuria and eGFR decline ${ }^{10}$. Yet CKD progression even among those without macroalbuminuria was still much 
greater than that expected for healthy aging ${ }^{10}$. It is crucial to identify the mechanisms that lead to the progression of kidney impairment beyond albuminuria in order to identify risk factors for CKD progression, in an effort to prevent further decline in kidney function. Therefore, this analysis of the eGFR Follow-up Study aims to assess whether cardio-metabolic risk markers of central obesity, hyperglycaemia, dyslipidaemia, hypertension and chronic inflammation were associated with CKD progression in Indigenous Australians with and without baseline albuminuria.

\section{Subjects and Methods}

\section{Participants}

The eGFR Baseline Study, an observational study of 654 Indigenous Australian men and women aged e 16 years, recruited participants between 2007 and 2011 from urban, rural and remote centres in Australia where Indigenous people experience high rates of ESKD ${ }^{11,12}$. Recruitment was across five pre-defined strata: (i) "healthy" people without diabetes, CKD or albuminuria, (ii) participants with physician diagnosed diabetes or albuminuria and eGFR (4 variable Modification in Diet of Renal Disease equation) $>90 \mathrm{ml} / \mathrm{min}^{\prime} 1.73 \mathrm{~m}^{2}$; (iii) eGFR $60-90 \mathrm{ml} / \mathrm{min} / 1.73 \mathrm{~m}^{2}$; (iv) eGFR30-59 $\mathrm{ml} / \mathrm{min}^{\prime} 1.73 \mathrm{~m}^{2}$; (v) eGFR $<15-29 \mathrm{ml} / \mathrm{min}^{\prime} 1.73 \mathrm{~m}^{2}$. Participants with CKD and/or diabetes were volunteers from participating medical services, and "healthy" participants were volunteers from the surrounding community. Individuals identified as having rapidly changing kidney function, receiving dialysis, pregnant or breastfeeding, or had an allergy or adverse reaction to iodine-based contrast media were not eligible.

Of the 654 baseline participants, 619 were eligible to participate in the observational eGFR Followup Study. Participants were not eligible if aged $<18$ years $(n=13)$, withdrew consent $(n=7)$ or had no 
baseline blood sample $(n=15){ }^{10}$. This analysis was based on 520 participants. This analysis excluded 99 participants who were: (i) lost to follow-up (n=8), (ii) acutely unwell at the follow-up examination ( $n=1$ ), (ii) examined $<6$ months after the baseline examination ( $n=14)$, (iii) missing enzymatic creatinine measures at follow-up ( $\mathrm{n}=46)$, and (iv) missing baseline uACR measurements $(n=30)$. Compared to participants who were excluded from this analysis, those included $(n=520)$ had an older mean (sd) age (46 [15] vs. 41 [15] years, $\mathrm{p}=0.0025)$, were equally likely to be men (37.5 vs. $34.3 \%, \mathrm{p}=0.55)$, more likely to have diabetes ( 43.7 vs. $32.0 \%, \mathrm{p}=0.031)$, equally likely to have macroalbuminuria $(21.0 v s .24 .2 \%, \mathrm{p}=0.49)$, had a greater mean (sd) body mass index (BMI) (30.5 [7.1] vs. 27.1 [6.9] kg/m², $\mathrm{p}<0.001)$ and similar systolic blood pressure (118 [18] vs. 118 [22] $\mathrm{mmHg}, \mathrm{p}=0.99)$. Participants provided informed consent, and the Human Research Ethics Committees of the joint Menzies School of Health Research—Northern Territory Department of Health Human Research Ethics Committee, including the Aboriginal subcommittee; Central Australian Human Research Ethics Committee; Western Australian Aboriginal Health Information and Ethics Committee, Royal Perth Measurements Hospital Ethics Committee and Cairns and Hinterland Health Services District Human Research Ethics Committee approved the study.

\section{Measurements}

At baseline and follow-up non-fasting venous blood samples were collected, and pathology and clinical records were reviewed ${ }^{10,11}$. In 369 (67\%) participants, serum creatinine was measured at baseline and follow-up using an IDMS- aligned enzymatic method (Roche Diagnostics, Australia) from thawed frozen sera $\left(-80^{\circ} \mathrm{C}\right)$ by a single laboratory (Melbourne Pathology, Melbourne Australia). For the remaining 181 participants who did not provide a follow-up blood sample 
measurements of serum creatinine were collected from IDMS-aligned laboratories local to each recruitment site. We estimated GFR using the Chronic Kidney Disease Epidemiology Collaboration equations (CKD-EPI eGFR) based on serum creatinine without use of the correction for African Americans ${ }^{13}$.

Accredited local laboratories provided baseline clinical data on $\mathrm{HbA}_{1 \mathrm{c}}$, urine creatinine and albumin (to determine urine albumin to creatinine ratio, ACR), C-reactive protein (using high sensitivity assays), high density lipoprotein (HDL) and total cholesterol ${ }^{11}$. Baseline anthropometric measurements of height, weight, fat free mass percentage from single frequency bioimpedance (ImpediMed, USA), waist and hip circumference were taken according to study protocol ${ }^{11}$. Seated blood pressure was measured three times and the mean was calculated (Welch Allyn Medical Products, Skaneateles Falls, USA) ${ }^{11}$. Medical records were reviewed to determine diabetes duration $(\mathrm{n}=119)$, prescription of HMG-CoA reductase inhibitor medicines (statins), angiotensin converting enzyme inhibitors (ACEI) and angiotensin II receptor antagonists (ARB), and glucose lowering medications at baseline. Data on use of anti-hypertensive medicines was restricted to ACEI and ARB classes as previous analysis of our work has shown that these medicines accounted for over $90 \%$ of all anti-hypertensive medicines taken by participants in this study ${ }^{14}$. Information regarding selfreported cigarette smoking status (current, ex-smoker and never smoked) was collected at baseline.

\section{Statistical analysis}

Baseline characteristics were described in terms of means and standard deviations (sd) for continuous variables, and numbers (proportions) for categorical variables according to the 2012 Kidney Disease Improving Global Outcomes (KDIGO) uACR classifications: (i) normoalbuminuria: 
uACR $<3 \mathrm{mg} / \mathrm{mmol}(\mathrm{n}=297)$, microalbuminuria: uACR 3-30 mg/mmol (n=114) and macroalbuminuria: uACR e $30 \mathrm{mg} / \mathrm{mmol}(\mathrm{n}=109){ }^{15}$. Differences between groups were compared using one-way analysis of covariance (ANOVA) for continuous characteristics and $\chi^{2}$ squared tests for categorical characteristics. Continuous risk factors were normally distributed, except for Creactive protein, which was transformed by taking the natural logarithm prior to analysis. Linear mixed regression models were used to estimate risk factor associations between baseline cardiometabolic risk factors and annual CKD-EPI eGFR change ( $\mathrm{ml} / \mathrm{min} / 1.73 \mathrm{~m}^{2} /$ year). Annual CKD-EPI eGFR change was calculated as (CKD-EPI eGFR at follow-up minus CKD-EPI eGFR at baseline)/follow-up time. The follow-up time was the date between baseline and follow-up serum creatinine measurement (range, 0.52-5.75 years). For participants who died or commenced renal replacement therapy, the most recent serum creatinine measurement was used before death or commencement of therapy, respectively, in the calculation of annual eGFR change. Models were adjusted for baseline age, sex and CKD-EPI eGFR. We also investigated the mean annual CKD-EPI eGFR decline according to both baseline albuminuria and glycaemia categories, and linear regression was used to assess trends in eGFR decline across glycaemic groups for those with normoalbuminuria. Glycaemia was classified as: (i) normal glycaemia: $\mathrm{HbA}_{\mathrm{lc}}<39 \mathrm{mmol} / \mathrm{mol}$ $(<5.7 \%)$ (n=129); (ii) intermediate hyperglycaemia: $\mathrm{HbA}_{1 \mathrm{c}}$ e 39 to $<48 \mathrm{mmol} / \mathrm{mol}$ (e $5.7 \%$ to $\left.<6.5 \%\right)$ ( $\mathrm{n}=159)$ and (iii) diabetes: $\mathrm{HbA}_{1 \mathrm{c}}$ e $48 \mathrm{mmol} / \mathrm{mol}$ (e $6.5 \%$ ) or physician-diagnosed type 2 diabetes $(n=227){ }^{16}$. Baseline characteristics of those with normal glycaemia, intermediate glycaemia and diabetes were compared among those with normoalbuminuria. Additionally, characteristics of those with normoalbuminuria, microalbuminuria and macroalbuminuria among those with diabetes were 
also compared. Analyses were conducted in Stata v14.1 (Stata Corporation, College Station, Texas, USA).

\section{Results}

Baseline characteristics according to baseline albuminuria

Follow-up was over a median of 3 years (inter-quartile range: $2.5-3.3$ ) and 1525 person-years. Mean annual change in CKD EPI eGFR $(95 \% \mathrm{CI})$ for the analysis population was -3.0 (-3.6 to -2.5$)$ $\mathrm{ml} / \mathrm{min} / 1.73 \mathrm{~m}^{2}$, and by baseline albuminuria categories as follows: -2.0 (-2.6 to- 1.4$),-2.5$ (-3.7 to $1.3)$ and $-6.3(-7.8$ to -4.9$) \mathrm{ml} / \mathrm{min} / 1.72 \mathrm{~m}^{2}$. Table 1 demonstrates that greater albuminuria was associated with older age and an adverse cardio-metabolic risk profile.

\section{Cardiometabolic associations with eGFR decline according to baseline albuminuria groups}

After adjusting for baseline eGFR levels and sex, older age was significantly associated with decline in eGFR in those with normoalbuminuria, but not in those with albuminuria. Although a difference in decline in eGFR between men and women of borderline statistical significance was noted in those with normoalbuminuria, this was not demonstrated in those with albuminuria (Table 2). Greater baseline blood pressure, C-reactive protein, waist-to-hip ratio, and lower HDL cholesterol showed non-significant trends with a decline in eGFR in the presence of albuminuria, but these relationships were reversed in those with normoalbuminuria, such that a worse risk factor profile tended to be associated with less eGFR decline, though wide confidence intervals precluded any firm conclusions on these trends. Moreover, while greater baseline $\mathrm{HbA}_{1 \mathrm{c}}$ showed a borderline association with eGFR decline ( $\mathrm{p}=0.059)$ among those with macroalbuminuria, this was not observed in those with 
microalbuminuria or normoalbuminuria. In fact, participants with normoalbuminuria and baseline diabetes experienced significantly less eGFR decline compared to those without diabetes and normoalbuminuria $(\mathrm{p}=0.027)$.

Figure 1 shows that the most pronounced pattern for eGFR decline according to worsening glycaemia was observed in participants with macroalbuminuria. eGFR decline according to glycaemic status was less consistent among those with microalbuminuria, and among those with normoalbuminuria eGFR decline was greater for those with normal glycaemia, compared to those with intermediate hyperglycaemia or diabetes ( $\mathrm{p}$ for trend $=0.014$ ).

eGFR decline in participants with baseline normoalbuminuria

To further understand the unexpected finding of greater eGFR decline among those with normal glycaemia compared to those with diabetes and normoalbuminuria, we described baseline characteristics according to baseline glycaemia among those with normoalbuminuria only. Although participants in this sub-group with diabetes had a worse risk factor profile at baseline in terms of older mean age, lower mean eGFR, higher systolic blood pressure and greater waist-to-hip ratio compared to those with normal glycaemia, those with diabetes had greater antihypertensive medication use than those without diabetes (Table 3). Furthermore, compared to those with diabetes and macroalbuminuria, participants with diabetes and normoalbuminuria had a more favourable baseline risk profile in terms of younger age ( 51 vs. 54 years), shorter duration of diabetes ( 8 vs. 11 years), lower mean systolic blood pressure (116 vs. $128 \mathrm{mmHg}$ ), lower mean $\mathrm{HbA}_{1 \mathrm{c}}$ (62 vs. 69 $\mathrm{mmol} / \mathrm{mol}$ or $7.8 \mathrm{vs} 8.5 \%$ ), greater eGFR (96 vs. $67 \mathrm{ml} / \mathrm{min} / 1.73 \mathrm{~m}^{2}$ ) and lower proportion taking both oral glucose lowering medication and insulin (13 vs 31\%), which suggests that these 
participants with diabetes and normoalbuminuria represent a group in the earlier phases of diabetic nephropathy.

\section{Discussion}

We recently reported that baseline albuminuria was powerfully predictive of eGFR decline at all levels of baseline eGFR among Indigenous Australian adults ${ }^{10}$. This is the first study to evaluate relationships between cardio-metabolic risk factors and eGFR decline according to albuminuria status in Indigenous Australians, and has highlighted that cardio-metabolic risk factors were not independent predictors of eGFR decline, but seem to exacerbate the decline in renal function in the setting of existing proteinuria. We observed a borderline association between greater baseline $\mathrm{HbA}_{1 \mathrm{c}}$ and eGFR decline in those with macroalbuminuria, but this was not observed in those with microalbuminuria or normoalbuminuria. While greater baseline blood pressure, C-reactive protein, waist-to-hip ratio and lower HDL cholesterol tended towards an association with eGFR decline in the presence of albuminuria, wide confidence intervals and multiple comparisons between groups precluded firm conclusions on these relationships. Although this cohort has a higher than expected eGFR decline than other similarly aged populations the mechanisms explaining eGFR decline over this short follow-up period in Indigenous Australians beyond albuminuria remain to be elucidated.

In our study no consistent relationship between greater baseline $\mathrm{HbA}_{1 \mathrm{c}}$ and decline in eGFR was observed across different levels of baseline albuminuria. Indeed, among participants with normoalbuminuria, greater eGFR decline was observed for those with normoglycaemia compared to those with intermediate glycaemia or diabetes. It is possible that the low number of $\mathrm{HbA}_{1 \mathrm{c}}$ values in the normal range (only one quarter of the study population had a normal range value $\left(\mathrm{HbA}_{1 \mathrm{c}}<39\right.$ 
$\mathrm{mmol} / \mathrm{mol}$ or $<5.7 \%$ ) limited our ability to find a difference in eGFR decline between those with elevated and normal $\mathrm{HbA}_{1 \mathrm{c}}$ among those without macroalbuminuria. Alternatively, as categorisation of glycaemia and albuminuria groups was not based on random allocation, other unknown factors could have confounded associations seen in our observational study. The lack of an association of $\mathrm{HbA}_{1 \mathrm{c}}$ with eGFR decline for participants without macroalbuminuria is in contrast to findings from a South Korean population which demonstrated a strong association between $\mathrm{HbA}_{1 \mathrm{c}}$ and eGFR among participants free of diabetes. However, that cross-sectional study did not assess the contribution of $\mathrm{HbA}_{1 \mathrm{c}}$ to progression of eGFR. Furthermore, we have shown diabetes to be significantly associated with renal disease outcomes ${ }^{10}$, and another large prospective study of a multi-ethnic US population has shown diabetes to be predictive of the development of $\mathrm{CKD}$, regardless of baseline albuminuria status ${ }^{17}$, but the CKD outcomes used in these studies related to treated $\mathrm{CKD}$ rather than eGFR decline. These associations between diabetes and renal outcomes may have been influenced by medical management decisions.

Divergent renal pathologies may be one explanation for the different patterns of eGFR decline in participants with and without albuminuria and with and without diabetes. For participants with normoalbuminuria at baseline, we showed a greater decline in eGFR for participants with normal glycaemia compared to participants with diabetes at baseline. The lack of eGFR decline in participants with diabetes and normoalbuminuria may be a result of the glomerular hyperfiltrating effects of glucose reabsorption as this group of participants had a relatively short period of diagnosed diabetes and well controlled blood pressure. Hyperfiltration has also been observed in the early stages of diabetic nephropathy in the PIMA Indian population ${ }^{18}$. These observations of glomerular hyperfiltration could also indicate a role for sodium glucose cotransporter 2 inhibitors (SGLT2i), 
which are a relatively new class of diabetes medicines that lowers plasma glucose by inhibiting glucose reabsorption by the glomeruli, but we were not able to assess this as the baseline examination of our study was undertaken before SGLT2i became available. Alternatively, as a high proportion of participants with diabetes were also prescribed anti-hypertensive medications, the observed attenuated eGFR decline could have also been explained by clinical management and effective therapeutic relationships that acted as potential confounders over this short follow-up. Further research with longer follow-up in a cohort with early onset diabetes and normoalbuminuria is required to explore the effects of hyperfiltration on eGFR decline and whether these effects occur in conjunction with metabolic risk factors, and act as a potential target for effective interventions.

The trends observed between greater baseline blood pressure and eGFR decline were only observed in those with macroalbuminuria. In those without macroalbuminuria there was a trend of less eGFR decline with greater blood pressure. However, a large proportion of our cohort, in particular those with albuminuria, were taking ACEI/ARB medicines, and this may have confounded the associations observed for blood pressure. Nevertheless, others show that the relationship between systolic blood pressure and kidney disease progression was mediated by urine protein level, whereby a graded relationship between greater systolic blood pressure and kidney disease progression was only evident among those high urine protein ${ }^{19}$. Furthermore, intervention studies have demonstrated that antihypertensive agents are effective in reducing mortality and progression of CKD ${ }^{20}$.

We did not observe strong associations between cardio-metabolic risk factors and eGFR decline in our study. However, trends of greater $\mathrm{HbA}_{1 \mathrm{c}}$, baseline blood pressure, C-reactive protein, waist-tohip ratio and lower HDL cholesterol with greater eGFR decline were only observed in the presence 
of albuminuria. These current findings are also supported by our previous work ${ }^{14}$, and that of others 4,21-23, which have demonstrated significant independent associations of diabetes, waist-to-hip ratio, and systolic blood pressure with albuminuria. Thus, due to their associations with albuminuria, these cardio-metabolic risk factors remain important targets for clinical interventions, and further research is required to investigate associations between these risk factors and change in albuminuria in our population. Nonetheless, our lack of significant associations between cardio-metabolic risk factors and decline in eGFR indicates that other mechanisms for eGFR decline in the absence of albuminuria may be operating. Early life experiences, including low birth weight, repeated infections, poststreptococcal glomerulonephritis and poor nutrition have been implicated with the development of albuminuria, as well as the amplification of relationships between cardio-metabolic risk factors and albuminuria ${ }^{22}$. However, as these factors were not measured in our study we were unable to assess the impact of this multidimensional model on eGFR decline, and further research of the impact of these other potential risk factors is warranted.

The eGFR Follow-up Study is the largest study to investigate predictors of eGFR decline in Indigenous Australians according to albuminuria status. Decline in eGFR was determined by a single laboratory using an IDMS-aligned enzymatic serum creatinine assay for the majority of participants, and there was minimal loss to follow-up ${ }^{10}$. Participant recruitment occurred in diverse geographical regions across Western Australia, Northern Territory and Queensland, thus these findings may also be generalisable to Indigenous Australians living in urban, rural and remote locations, as well as those with and without diabetes and kidney disease.

Nevertheless, limitations exist. First, the observations in this study are based on a short follow-up 
period and it is not known whether the relationships between cardio-metabolic predictors and eGFR decline might be different with longer follow-up. Second, participants were not randomly selected and therefore the representativeness of the study population to the target population of Indigenous Australians at high risk of ESKD is unknown. Third, although we have previously demonstrated that the CKD-EPI eGFR equation can predict measured GFR well in this population ${ }^{12}$, our data were based on single measures and thus the impact of individual variability of serum creatinine, and that of urine creatinine and albumin could not be assessed and may have attenuated our findings. Fourth, although the mean eGFR decline among those without macroalbuminuria was larger than -1.0 $\mathrm{ml} / \mathrm{min} / 1.73 \mathrm{~m}^{2} /$ year expected for ageing ${ }^{15}$, declines in eGFR among those with normoalbuminuria ($\left.2.0 \mathrm{ml} / \mathrm{min} / 1.73 \mathrm{~m}^{2}\right)$ and microalbuminuria $\left(-2.5 \mathrm{ml} / \mathrm{min} / 1.73 \mathrm{~m}^{2}\right)$ were smaller than that observed with macroalbuminuria $\left(-6.3 \mathrm{ml} / \mathrm{min} / 1.73 \mathrm{~m}^{2}\right)$, and this may have limited our ability to observe significant relationships between the cardio-metabolic predictors and eGFR decline in those without overt albuminuria. Fifth, we acknowledge the limitation of multiple testing in this study, and caution should be taken when interpreting results that have a $\mathrm{p}$ value of borderline significance. Finally, we cannot assess the impact of pharmacological treatment on our findings, as medicine use was not based on random allocation but medical condition status at baseline.

In conclusion, over three years our observational study showed marked eGFR decline with greater baseline albuminuria. While significant associations were not observed between cardio-metabolic risk factors and eGFR decline, observed trends indicated that cardio-metabolic risk factors tended to be related to a decline in eGFR in the presence of albuminuria. Specifically, the association between greater baseline hyperglycaemia and eGFR decline among individuals with baseline macroalbuminuria was lacking for participants without macroalbuminuria. These findings are 
challenging to the current conceptualised framework of CKD in Indigenous Australians. While it is acknowledged that macroalbuminuria with impaired glycaemia are critically associated with eGFR decline, there is presently an evidence gap in explaining eGFR decline in adults without macroalbuminuria and without diabetes. The activation of other pathways that are not captured by the clinical and biochemical parameters measured in this study, such as those linked to early life experiences, post-streptococcal glomerulonephritis and inflammation may also play a role in promoting GFR loss in Indigenous Australians, and further research to understand their potential role in kidney disease is required.

Words: 3321

\section{Acknowledgements}

The authors gratefully acknowledge the support of the eGFR Study participants, study staff, and partner organizations. We thank Dr. Kevin Warr and Dr. William Majoni for facilitating participant recruitment and follow-up at the sites of their employing organization and Loyla Leysley, Sian Graham, Mary Ward, and Joseph Fitz for assistance with follow-up in their communities. The authors also thank Melbourne Pathology for providing the technical support in the enzymatic creatinine analysis and Roche Diagnostics for supplying the enzymatic creatinine reagent kit for the baseline study.

The eGFR Study was funded by the National Health and Medical Research Council of Australia (NHMRC, Project Grants \#545202 and \#1021460), with additional support from Kidney Health Australia, NHMRC Program Grant (\#631947), the Colonial Foundation, Diabetes Australia Research Trust, Rebecca L Cooper Foundation and SeaSwift, Thursday Island. ELMB was supported by a 
National Health and Medical Research Council (NHMRC) Training Research Fellowship (APP1016612); LJM-B was supported by a NHMRC Fellowship (\#605837) and NHMRC Practitioner Fellowship (\#1078477); FB was supported by NHMRC Program Grant (\#631947); JTH was supported by NHMRC Fellowship \#1092576 and RACP Jacquot Research Establishment Award; PDL was supported by NHMRC Scholarship \#1038721 and RACP Jacquot Research Establishment Award; EIE was supported by an NHMRC Early Career Fellowship: Health Professional Research Fellowship (Part Time, \#1054312), Viertel Clinical Investigatorship and a RACP JDRF Research Establishment Fellowship. WH directs the NHMRC funded Centre for Research Excellence in Chronic Kidney Disease (\#1079502). ADHB is supported by a Viertel Senior Medical Research Fellowship. RM was supported by an Australian Diabetes Society-Servier Diabetes Research Grant, Rebecca Cooper Medical Research Foundation and the St Vincent's Hospital Melbourne Research Endowment Fund. The views expressed in this publication are those of the authors and do not reflect the views of the NHMRC. Funding bodies had no role in the study design, in the collection, analysis or interpretation of data, in the writing of the manuscript or the decision to submit the manuscript for publication.

\section{eGFR Study investigators}

LJ Maple-Brown, PD Lawton, WE Hoy, A Cass, G Jerums, RJ MacIsaac, L Ward, M Thomas, K O’Dea, J Hughes, A Sinha, R MacDermott, G Jones, A Ellis, LS Piers, K Warr, A Brown, S Cherian and W Majoni.

Conflict of Interest statement: None of the authors have any disclosures. 


\section{References}

1 Australian Institute of Health and Welfare. Chronic kidney disease in Aboriginal and Torres Strait Islander People 2011. Cat. no. PHE 151. Canberra: AIHW 2011.

2 McDonald SP. End-stage kidney disease among indigenous peoples of Australia and New Zealand. Kidney international supplements. 2013; 3: 170-73.

3 Cass A, Cunningham J, Snelling P, Wang Z, Hoy W. End-stage renal disease in indigenous Australians: a disease of disadvantage. Ethn Dis. 2002; 12: 373-8.

4 Hoy WE, Mathews JD, McCredie DA, Pugsley DJ, Hayhurst BG, Rees M, et al. The multidimensional nature of renal disease: rates and associations of albuminuria in an Australian Aboriginal community. Kidney international. 1998; 54: 1296-304.

5 Gansevoort RT, Matsushita K, van der Velde M, Astor BC, Woodward M, Levey AS, et al. Lower estimated GFR and higher albuminuria are associated with adverse kidney outcomes. A collaborative meta-analysis of general and high-risk population cohorts. Kidney international. 2011; 80: 93-104.

6 Minges KE, Zimmet P, Magliano DJ, Dunstan DW, Brown A, Shaw JE. Diabetes prevalence and determinants in Indigenous Australian populations: A systematic review. Diabetes Res Clin Pract. 2011; 93: 139-49.

7 Hoy WE, Samuel T, Mott SA, Kincaid-Smith PS, Fogo AB, Dowling JP, et al. Renal biopsy findings among Indigenous Australians: a nationwide review. Kidney international. 2012; 82: 1321-31.

8 Samuel SM, Palacios-Derflingher L, Tonelli M, Manns B, Crowshoe L, Ahmed SB, et al. Association between First Nations ethnicity and progression to kidney failure by presence and severity of albuminuria. CMAJ. 2014; 186: E86-94. 
9 Hoy WE, Wang Z, VanBuynder P, Baker PR, McDonald SM, Mathews JD. The natural history of renal disease in Australian Aborigines. Part 2. Albuminuria predicts natural death and renal failure. Kidney international. 2001; 60: 249-56.

10 Maple-Brown LJ, Hughes JT, Ritte R, Barzi F, Hoy WE, Lawton PD, et al. Progression of Kidney Disease in Indigenous Australians: The eGFR Follow-up Study. Clinical journal of the American Society of Nephrology : CJASN. 2016; 11: 993-1004.

11 Maple-Brown LJ, Lawton PD, Hughes JT, Sharma SK, Jones GR, Ellis AG, et al. Study Protocol--accurate assessment of kidney function in Indigenous Australians: aims and methods of the eGFR study. BMC public health. 2010; 10: 80.

12 Maple-Brown LJ, Hughes JT, Lawton PD, Jones GR, Ellis AG, Drabsch K, et al. Accurate assessment of kidney function in indigenous Australians: the estimated GFR study. American journal of kidney diseases : the official journal of the National Kidney Foundation. 2012; 60: 6802.

13 Inker LA, Schmid CH, Tighiouart H, Eckfeldt JH, Feldman HI, Greene T, et al. Estimating glomerular filtration rate from serum creatinine and cystatin C. $N$ Engl J Med. 2012; 367: 20-9.

14 Hughes J, Maple-Brown L, Thomas M, Lawton P, Sinha A, Cass A, et al. Cross-sectional associations of albuminuria among Aboriginal and Torres Strait Islander adults: the eGFR Study. Nephrology. 2016.

15 Group. KDIGOKCW. KDIGO 2012 clinical practice guideline for the evaluation and management of chronic kidney disease. Kidney international Supplement. 2013; 3: 1-150.

16 American Diabetes A. Diagnosis and classification of diabetes mellitus. Diabetes Care. 2011; 34 Suppl 1: S62-9.

17 Chang TI, Li S, Chen SC, Peralta CA, Shlipak MG, Fried LF, et al. Risk factors for ESRD in individuals with preserved estimated GFR with and without albuminuria: results from the 
Kidney Early Evaluation Program (KEEP). American journal of kidney diseases : the official journal of the National Kidney Foundation. 2013; 61: S4-11.

18 Nelson RG, Bennett PH, Beck GJ, Tan M, Knowler WC, Mitch WE, et al. Development and progression of renal disease in Pima Indians with non-insulin-dependent diabetes mellitus. Diabetic Renal Disease Study Group. N Engl J Med. 1996; 335: 1636-42.

19 Jafar TH, Stark PC, Schmid CH, Landa M, Maschio G, de Jong PE, et al. Progression of chronic kidney disease: the role of blood pressure control, proteinuria, and angiotensinconverting enzyme inhibition: a patient-level meta-analysis. Annals of internal medicine. 2003; 139: $244-52$.

20 Hoy WE, Wang Z, Baker PR, Kelly AM. Reduction in natural death and renal failure from a systematic screening and treatment program in an Australian Aboriginal community. Kidney international Supplement. 2003: S66-73.

21 Hoy WE, Wang Z, VanBuynder P, Baker PR, Mathews JD. The natural history of renal disease in Australian Aborigines. Part 1. Changes in albuminuria and glomerular filtration rate over time. Kidney international. 2001; 60: 243-8.

22 Hoy WE, White AV, Tipiloura B, Singh G, Sharma SK, Bloomfield H, et al. The multideterminant model of renal disease in a remote Australian Aboriginal population in the context of early life risk factors: lower birth weight, childhood post-streptococcal glomerulonephritis, and current body mass index influence levels of albuminuria in young Aboriginal adults. Clinical nephrology. 2015; 83: 75-81.

23 Nelson RG, Knowler WC, Pettitt DJ, Hanson RL, Bennett PH. Incidence and determinants of elevated urinary albumin excretion in Pima Indians with NIDDM. Diabetes Care. 1995; 18: 182-7. 
Words: 798

This article is protected by copyright. All rights reserved. 
Table 1. Baseline characteristics according to baseline albuminuria status: the eGFR study

\begin{tabular}{|c|c|c|c|c|c|}
\hline & $\begin{array}{c}\text { Normoalbuminuria } \\
(\mathrm{n}=\mathbf{2 9 7})\end{array}$ & $\begin{array}{c}\text { Microalbuminuria } \\
(n=114)\end{array}$ & $\begin{array}{c}\text { Macroalbuminuria } \\
(\mathrm{n}=109)\end{array}$ & P value & $\begin{array}{c}\text { Total } \\
(\mathbf{n}=\mathbf{5 2 0})\end{array}$ \\
\hline Age (years) & $42(14)$ & $51(14)$ & $51(13)$ & $<0.001$ & $46(15)$ \\
\hline Men $(\%)$ & $109(36.7)$ & $38(33.3)$ & $48(44.0)$ & 0.23 & $195(37.5)$ \\
\hline Serum creatinine $(\mu \mathrm{mol} / \mathrm{L})$ & $69.1(17.4)$ & $78.6(37.6)$ & $128.3(87.7)$ & $<0.001$ & $83.4(51.0)$ \\
\hline CKD-EPI eGFR $\left(\mathrm{ml} / \mathrm{min} / 1.73 \mathrm{~m}^{2}\right)^{\dagger}$ & $102(17.8)$ & $90.2(28.1)$ & $66.4(35.7)$ & $<0.001$ & $91.9(28.5)$ \\
\hline Weight $(\mathrm{kg})$ & $84.7(20.9)$ & $86.9(21.7)$ & $83.6(22.7)$ & 0.50 & $85.0(21.5)$ \\
\hline Body mass index $\left(\mathrm{kg} / \mathrm{m}^{2}\right)$ & $30.2(6.9)$ & $31.6(7.5)$ & $30.1(7.5)$ & 0.18 & $30.5(7.1)$ \\
\hline Waist circumference $(\mathrm{cm})$ & $100.3(16.2)$ & $104.2(15.7)$ & $104.9(16.2)$ & 0.016 & $102.1(16.2)$ \\
\hline Waist-hip-ratio & $0.93(0.084)$ & $0.96(0.092)$ & $0.99(0.094)$ & $<0.001$ & $0.95(0.092)$ \\
\hline Fat free mass $(\%)$ & $65.5(9.4)$ & $63.0(8.2)$ & $65.3(9.4)$ & 0.063 & $64.9(9.2)$ \\
\hline Diabetes $(\%)$ & $80(26.9)$ & $69(60.5)$ & $78(71.6)$ & $<0.001$ & $227(43.7)$ \\
\hline Haemoglobin $\mathrm{A}_{\mathrm{lc}}(\mathrm{mmol} / \mathrm{ml})$ & 45. (15.1) & $56.9(22.5)$ & $60.7(23.1)$ & $<0.001$ & $50.8(20.0)$ \\
\hline Haemoglobin $\mathrm{A}_{1 \mathrm{c}}(\%)$ & $6.3(1.39)$ & $7.36(2.06)$ & $7.71(2.11)$ & $<0.001$ & $6.80(1.83)$ \\
\hline Current smoker $(\%)$ & $131(44.7)$ & $40(35.7)$ & $33(31.1)$ & 0.030 & $204(39.9)$ \\
\hline Systolic blood pressure (mmHg) & $114(15)$ & $120(18)$ & $127(19)$ & $<0.001$ & $118(18)$ \\
\hline Diastolic blood pressure (mmHg) & $73(10)$ & $76(10)$ & $77(12)$ & $<0.001$ & $75(10)$ \\
\hline Total cholesterol $(\mathrm{mmol} / \mathrm{L})$ & $5.0(1.03)$ & $4.71(1.08)$ & $4.48(1.04)$ & $<0.001$ & $4.81(1.06)$ \\
\hline HDL cholesterol $(\mathrm{mmol} / \mathrm{L})^{\ddagger}$ & $1.11(0.36)$ & $1.04(0.25)$ & $1.03(0.34)$ & 0.045 & $1.08(0.34)$ \\
\hline Total cholesterol/HDL Ratio & $4.83(1.58)$ & $4.76(1.40)$ & $4.72(1.61)$ & $<0.001$ & $4.79(1.54)$ \\
\hline $\begin{array}{l}\text { HMG-CoA reductase inhibitor } \\
\text { medicine use }(\%)\end{array}$ & $45(32)$ & $38(27)$ & $58(41)$ & $<0.001$ & $141(27)$ \\
\hline C-reactive protein $(\mathrm{mg} / \mathrm{L})$ & $5.5(3,10)$ & $6.4(3,13.5)$ & $5.9(3,11)$ & 0.83 & $6.0(3,11)$ \\
\hline $\begin{array}{l}\text { Angiotensin converting enzyme } \\
\text { inhibitor medicine use }(\%)\end{array}$ & $43(14.5)$ & $50(43.9)$ & $62(56.9)$ & $\begin{array}{l}0.20 \\
<0.001\end{array}$ & $155(29.8)$ \\
\hline $\begin{array}{l}\text { Angiotensin II receptor antagonist } \\
\text { medicine use }(\%)\end{array}$ & $14(4.7)$ & $13(11.4)$ & $27(24.8)$ & $<0.001$ & $54(10.4)$ \\
\hline
\end{tabular}

Data are mean (sd), median $\left(25^{\text {th }}, 75^{\text {th }}\right.$ percentile) or number (\%). ${ }^{7}$ CKD-EPI eGFR: Chronic Kidney on creatinine without the African American correction; ${ }^{\sharp} \mathrm{HDL}$ : high density lipoprotein cholesterol. 
Table 2: Relationship of the annual absolute decline in eGFR (CKD EPI) with participant characteristics at baseline according to baseline albuminuria

\begin{tabular}{|c|c|c|c|c|c|c|}
\hline & \multicolumn{2}{|l|}{$\begin{array}{r}\text { Normoalbuminuria } \\
(\mathrm{n}=297)\end{array}$} & \multicolumn{2}{|l|}{$\begin{array}{r}\text { Microalbuminuria } \\
(n=114)\end{array}$} & \multicolumn{2}{|l|}{$\begin{array}{r}\text { Macroalbuminuria } \\
(n=109)\end{array}$} \\
\hline & coefficient & p value & coefficient & p value & coefficient & p value \\
\hline Age (per 5 years) & $-0.36(-0.62$ to -0.10$)$ & 0.007 & $-0.45(-1.03$ to 0.12$)$ & 0.121 & $0.51(-0.14$ to 1.15$)$ & 0.124 \\
\hline Women & $1.18(-0.04$ to 2.40$)$ & 0.058 & $0.34(-2.20$ to 2.87$)$ & 0.793 & $-0.15(-3.04$ to 2.75$)$ & 0.919 \\
\hline Weight (per $5 \mathrm{~kg}$ ) & $-0.06(-0.20$ to 0.08$)$ & 0.4 & $0.24(-0.05$ to 0.52$)$ & 0.108 & $0.20(-0.13$ to 0.54$)$ & 0.227 \\
\hline Body mass index (per $\mathrm{kg} / \mathrm{m}^{2}$ ) & $-0.02(-0.11$ to 0.06$)$ & 0.59 & $0.16(0.00$ to 0.33$)$ & 0.049 & $0.12(-0.08$ to 0.32$)$ & 0.234 \\
\hline Waist circumference (per $2 \mathrm{~cm}$ ) & $0.01(-0.07$ to 0.09$)$ & 0.771 & $0.07(-0.10$ to 0.24$)$ & 0.417 & $0.08(-0.10$ to 0.26$)$ & 0.367 \\
\hline Waist-to-hip ratio (0.1 unit) & $7.62(-0.26$ to 15.50$)$ & 0.058 & $-8.84(-25.25$ to 7.57$)$ & 0.288 & $0.03(-17.46$ to 17.52$)$ & 0.997 \\
\hline Fat free mass (\%) & $-0.028(-0.13$ to 0.71$)$ & 0.57 & $-0.11(-0.31$ to 0.089$)$ & 0.27 & $-0.045(-0.27$ to 0.178$)$ & 0.69 \\
\hline Diabetes ( $v s$ no diabetes) & $1.60(0.18$ to 3.01$)$ & 0.027 & $-0.36(-2.83$ to 2.11$)$ & 0.774 & $-2.76(-6.07$ to 0.56$)$ & 0.102 \\
\hline Haemoglobin $\mathrm{A}_{1 \mathrm{c}}($ per $1 \mathrm{mmol} / \mathrm{mol})$ & $0.025(-0.015$ to 0.067$)$ & 0.23 & $0.036(-0.018$ to 0.092$)$ & 0.187 & $-0.063(-0.13$ to 0.0025$)$ & 0.059 \\
\hline Haemoblobin $\mathrm{A}_{1 \mathrm{c}}($ per $1 \%)$ & $0.28(-0.17$ to 0.73$)$ & 0.228 & $0.40(-0.20$ to 1.01$)$ & 0.187 & $-0.69(-1.41$ to 0.03$)$ & 0.059 \\
\hline Currently smoking & $0.81(-0.42$ to 2.04$)$ & 0.197 & $-0.78(-3.56$ to 2.01$)$ & 0.582 & $2.17(-1.12$ to 5.47$)$ & 0.194 \\
\hline Systolic blood pressure (per $5 \mathrm{mmHg}$ ) & $0.07(-0.14$ to 0.27$)$ & 0.514 & $0.14(-0.22$ to 0.49$)$ & 0.452 & $-0.10(-0.49$ to 0.30$)$ & 0.627 \\
\hline Diastolic blood pressure (per $5 \mathrm{mmHg}$ ) & $0.11(-0.19$ to 0.42$)$ & 0.475 & $0.51(-0.09$ to 1.12$)$ & 0.097 & $-0.09(-0.74$ to 0.56$)$ & 0.779 \\
\hline Total cholesterol (per $1 \mathrm{mmol} / \mathrm{l})$ & $0.38(-0.19$ to 0.95$)$ & 0.188 & $0.96(-0.21$ to 2.14$)$ & 0.106 & $1.42(-0.05$ to 2.88$)$ & 0.058 \\
\hline HDL cholesterol (per $1 \mathrm{mmol} / \mathrm{l}^{\dagger}$ & $-0.93(-2.57$ to 0.71$)$ & 0.267 & $4.29(-0.96$ to 9.54$)$ & 0.108 & $2.48(-2.13$ to 7.09$)$ & 0.288 \\
\hline Total cholesterol to HDL ratio (per 0.1 unit) & $0.31(-0.07$ to 0.69$)$ & 0.109 & $-0.14(-1.07$ to 0.79$)$ & 0.76 & $0.40(-0.59$ to 1.39$)$ & 0.426 \\
\hline C-reactive protein (per $1 \log \mathrm{mg} / \mathrm{L}$ ) & $0.62(0.00$ to 1.25$)$ & 0.052 & $0.32(-0.74$ to 1.37$)$ & 0.555 & $-0.67(-2.15$ to 0.80$)$ & 0.369 \\
\hline ACEI or ARB use ( $v s$ no ACEI/ARB) $)^{\ddagger}$ & $0.25(-1.39$ to 1.89$)$ & 0.765 & $0.20(-2.31$ to 2.71$)$ & 0.875 & $-1.80(-4.88$ to 1.28$)$ & 0.25 \\
\hline
\end{tabular}

Data are coefficient $(95 \% \mathrm{CI})$ from linear regression models adjusted for age, sex and baseline Chronic Kidney Disease Epidemiological Collaboration estimated Glomerular Filtration Rate

equation based on creatinine (CKD-EPI eGFR). Albuminuria categories were: Normoalbuminuria $<27 \mathrm{mg} / \mathrm{g}$ (3mg/mmol); Microalbuminuria 27-265 mg/g (3-30 mg/mmol); Macroalbuminuria

$>265 \mathrm{mg} / \mathrm{g}(30 \mathrm{mg} / \mathrm{mmol}) .{ }^{\dagger} \mathrm{HDL}$ : high-density lipoprotein cholesterol; ${ }^{\star} \mathrm{ACEI}$, angiotensin converting enzyme inhibitor medication use; ARB, angiotensin II receptor blocker medication use.

This article is protected by copyright. All rights reserved. 
Table 3: Baseline characteristics of participants according to glucose tolerance status among those with normoalbuminuria at baseline: the eGFR study

\begin{tabular}{|c|c|c|c|}
\hline Baseline characteristics & Normal glycaemia & Intermediate glycaemia & Diabetes \\
\hline $\mathrm{n}$ & 98 & 117 & 80 \\
\hline Age (years) & $35(12)$ & $42(14)$ & $51(11)$ \\
\hline CKD-Epi eGFR $\left(\mathrm{ml} / \mathrm{min} / 1.73 \mathrm{~m}^{2}\right)^{\dagger}$ & $106.4(17.0)$ & $100.9(17.1)$ & $95.8(18.2)$ \\
\hline Women $(\%)$ & $60(61)$ & $74(63)$ & $53(66)$ \\
\hline Systolic blood pressure (mmHg) & $113(15)$ & $113(14)$ & $116(16)$ \\
\hline Waist-to-hip ratio & $0.89(0.08)$ & $0.92(0.08)$ & $0.97(0.08)$ \\
\hline C-reactive protein $(\mathrm{mg} / \mathrm{L})$ & $4.0(1.7,8.2)$ & $6.2(3.5,11.0)$ & $5.3(3.0,12.0)$ \\
\hline ACEI or ARB use $(\%)^{\ddagger}$ & $5(5)$ & $13(11)$ & $38(48)$ \\
\hline Diabetes duration (years) $)^{\S}$ & - & - & $8(3,13)$ \\
\hline \multicolumn{4}{|l|}{ Glucose lowering medication $(\%)^{\S}$} \\
\hline - $\quad$ No medication / diet only & - & - & $23(33)$ \\
\hline - $\quad$ Oral only & - & - & $37(54)$ \\
\hline - $\quad$ Oral and/or insulin & - & - & $9(13)$ \\
\hline
\end{tabular}

Data are mean (sd), median (25th, 75 th percentile) or number (\%).

${ }^{\dagger}$ CKD-EPI eGFR: Chronic Kidney Disease Epidemiological Collaboration estimated Glomerular Filtration Rate equation based on creatinine without the African American correction;

${ }^{\ddagger} \mathrm{ACEI}$, angiotensin converting enzyme inhibitor medication use; ARB, angiotensin II receptor blocker medication use

${ }^{\S}$ Information on diabetes duration and medication apply only to participants with physician-diagnosed type 2 diabetes $(\mathrm{n}=69)$.

Glycaemia was classified as: (i) normal glyaemia: $\mathrm{HbA}_{\mathrm{lc}}<39 \mathrm{mmol} / \mathrm{mol}\left(<5.7 \%\right.$ ), (ii) intermediate hyperglycaemia: $\mathrm{HbA}_{1 \mathrm{c}}$ e 39 to $<48 \mathrm{mmol} / \mathrm{mol}(\mathrm{e} 5.7 \%$ to $<6.5 \%$ ) and (iii) diabetes: $\mathrm{HbA} 1 \mathrm{c}$

e $48 \mathrm{mmol} / \mathrm{mol}(\mathrm{e} 6.5 \%)$ or physician-diagnosed type 2 diabetes [18]

This article is protected by copyright. All rights reserved. 


\section{Figure legends}

Figure 1: Absolute decline in eGFR according to baseline $\mathrm{HbA}_{1 \mathrm{c}}$ and albuminuria groups

Notes: The size of the boxes is proportional to the sample size (n). Albuminuria classified according to the 2012 Kidney Disease Improving Global Outcomes (KDIGO) uACR classifications: (i) normoalbuminuria: $\mathrm{uACR}<3 \mathrm{mg} / \mathrm{mmol}(\mathrm{n}=295)$, microalbuminuria: uACR 3-30 mg/mmol ( $\mathrm{n}=112)$ and macroalbuminuria: uACR e $30 \mathrm{mg} / \mathrm{mmol}(\mathrm{n}=108)^{15}$. Glycaemia was classified as: (i) normal glyaemia: $\mathrm{HbA}_{1 \mathrm{c}}<39 \mathrm{mmol} / \mathrm{mol}(<5.7 \%)(\mathrm{n}=129)$; (ii) intermediate hyperglycaemia: $\mathrm{HbA}_{1 \mathrm{c}}$ e 39 to $<48 \mathrm{mmol} / \mathrm{mol}(\mathrm{e} 5.7 \%$ to $<6.5 \%)(\mathrm{n}=159)$ and (iii) diabetes: $\mathrm{HbA}_{1 \mathrm{c}}$ e $48 \mathrm{mmol} / \mathrm{mol}(\mathrm{e} 6.5 \%$ ) or physician-diagnosed type 2 diabetes $(\mathrm{n}=227)^{16}$. Data in this Figure 1 is based on 515 participants with complete data for both albuminuria and diabetes status. Differences in eGFR decline between glycaemic groups for those with normoalbuminuria were significant ( $\mathrm{p}$ for trend $=0.014$ ). Non-linear relationships were observed between glycaemic status and eGFR decline for the microalbuminuria and macroalbuminuria groups and this prevented formal statistical assessment of linear trends. 
n $\mathrm{HbA}_{10}$ median $(\mathrm{QQR}) \mathrm{mmol} / \mathrm{mol}$ Mean $(95 \% \mathrm{Cl})$ annual change in eGFR $\mathrm{ml} / \mathrm{min} / 1.73 \mathrm{~m}^{2} /$ year

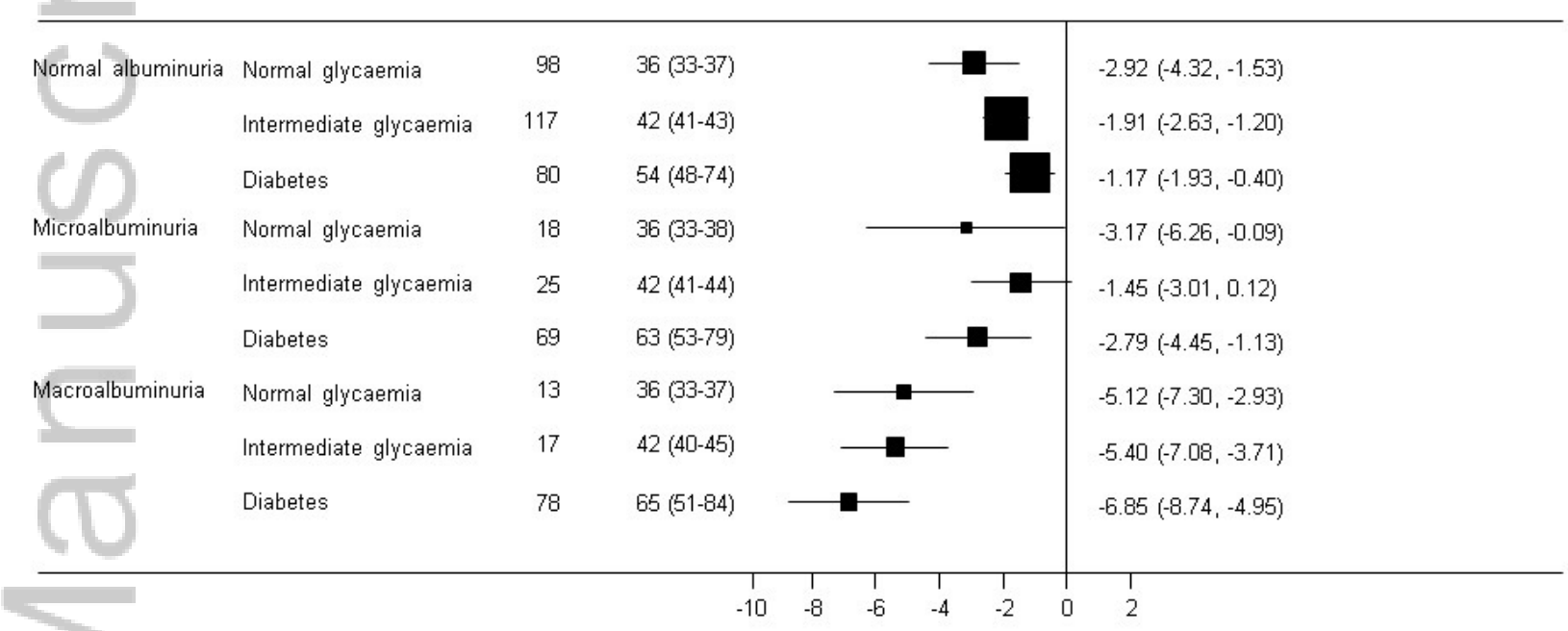

NEP_13073_F1.jpg

This article is protected by copyright. All rights reserved. 


\section{University Library}

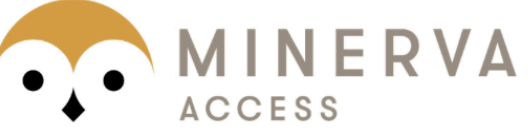

A gateway to Melbourne's research publications

Minerva Access is the Institutional Repository of The University of Melbourne

Author/s:

Barr, ELM;Barzi, F;Hughes, JT;Jerums, G;O'Dea, K;Brown, ADH;Ekinci, El;Jones, GRD;Lawton, PD;Sinha, A;Maclsaac, RJ;Cass, A;Maple-Brown, LJ

Title:

Contribution of cardiometabolic risk factors to estimated glomerular filtration rate decline in Indigenous Australians with and without albuminuria - the eGFR Follow-up Study

Date:

2018-07-01

Citation:

Barr, E. L. M., Barzi, F., Hughes, J. T., Jerums, G., O'Dea, K., Brown, A. D. H., Ekinci, E. I., Jones, G. R. D., Lawton, P. D., Sinha, A., Maclsaac, R. J., Cass, A. \& Maple-Brown, L. J. (2018). Contribution of cardiometabolic risk factors to estimated glomerular filtration rate decline in Indigenous Australians with and without albuminuria - the eGFR Follow-up Study. NEPHROLOGY, 23 (7), pp.682-689. https://doi.org/10.1111/nep.13073.

Persistent Link:

http://hdl.handle.net/11343/283418 